\title{
ON A CONJECTURE OF GRAHAM
}

\author{
J. W. SANDER
}

(Communicated by Larry T. Goldstein)

\begin{abstract}
Let $a_{1}<a_{2}<\cdots<a_{n}$ be a finite sequence of positive integers containing a prime power $p^{d}$ with the property: $a_{i} \neq p^{k} a_{j}$ for all $i, j$ and $k>0$. Then $\max _{i, j} a_{i} /\left(a_{i}, a_{j}\right) \geq n$.
\end{abstract}

In [4] R. L. Graham asks if the following is true: Let $A$ be a finite sequence of $n$ positive integers $a_{1}<a_{2}<\cdots<a_{n}$. Then $\max _{i, j} a_{i} /\left(a_{i}, a_{j}\right) \geq n$.

Several partial results have been obtained (see the references), particularly for the case where one of the $a_{i}$ 's is prime. The proofs for the latter follow similar lines, E. Z. Chein [2] and R. Klein [5] use G. Weinstein's improvement [9] upon R. Winterle's earlier result [10] while R. J. Simpson [7] can do without. In this paper we use refinements of the methods of Winterle [10] and Klein [5] to prove the following theorem. The sequence $A$ is said to be $p$-simple for a prime $p$ if $a_{i} \neq p^{k} a_{j}$ for $1 \leq i, j \leq n, k>0$.

THEOREM. Let $A$ be $p$-simple for a prime $p \neq 2$ and let $A$ contain a prime power $a_{k}=p^{d}(d \geq 0)$. Then

$$
\max _{1 \leq i, j \leq n} \frac{a_{i}}{\left(a_{i}, a_{j}\right)} \geq n
$$

In order to prove the theorem we first consider the case where $a_{1}$ is the required prime power. For a given sequence $A$ let $A=\left\{a_{1}, \ldots, a_{n}\right\}$.

LEMMA. Let $A$ be $p$-simple with $a_{1}=p^{d}(d \geq 0)$. Then

$$
\max _{i, j} \frac{a_{i}}{\left(a_{i}, a_{j}\right)} \geq n \text {. }
$$

PROOF. We may assume $d>0$, since the lemma is trivial for $a_{1}=1$. We suppose

$$
\max _{i, j} \frac{a_{i}}{\left(a_{i}, a_{j}\right)} \leq n-1
$$

This implies

$$
a_{n} \leq(n-1)\left(a_{n}, a_{1}\right) \leq(n-1) p^{d} .
$$

For $k=0,1, \ldots, d-1$ define

$$
B_{k}=\left\{a_{i} \in A: p^{k} \| a_{i}\right\}, \quad \text { and } \quad B=A \backslash \bigcup_{k=0}^{d-1} B_{k} .
$$

Received by the editors July 18, 1986 and, in revised form, November 5, 1986.

1980 Mathematics Subject Classification (1985 Revision). Primary 10A05; Secondary 02A05. 
Furthermore, for $k=0,1, \ldots, d-1$ and each $a_{i} \in B_{k}$ define

$$
T_{k}\left(a_{i}\right)=p^{h_{i, k}}\left(a_{i}-p^{k+1}\right)
$$

such that $h_{i, k}$ is maximal with

$$
T_{k}\left(a_{i}\right) \leq(n-1) p^{d} .
$$

Obviously, $T_{k}\left(a_{i}\right)>0$ and the maximality of $h_{i, k}$ gives

$$
T_{k}\left(a_{i}\right)>(n-1) p^{d-1} \text {. }
$$

We have for $a_{i} \in B_{k}$

$$
a_{i} \leq(n-1) p^{k} ;
$$

otherwise $a_{i}=p^{k} a_{i}^{\prime}$ with $a_{i}^{\prime} \geq n, p \nmid a_{i}^{\prime}$. This yields $a_{i} /\left(a_{i}, a_{1}\right)=p^{k} a_{i}^{\prime} / p^{k}=a_{i}^{\prime} \geq n$ which contradicts (1). By (3), (4) and (6) we have $h_{i, k} \geq d-k$ which implies

$$
p^{d} \mid T_{k}\left(a_{i}\right) \text {. }
$$

Since $\left(a_{i}, a_{i}-p^{k+1}\right)=\left(a_{i}, p^{k+1}\right)=p^{k}$ for $a_{i} \in B_{k}$, we get

$$
\left(a_{i}, T_{k}\left(a_{i}\right)\right)=p^{k} \text {. }
$$

We claim for $a_{i} \in B_{k}, a_{j} \in B$,

$$
T_{k}\left(a_{i}\right) \neq a_{j}
$$

otherwise by (5) and (8)

$$
a_{j} /\left(a_{j}, a_{i}\right)=T_{k}\left(a_{i}\right) /\left(T_{k}\left(a_{i}\right), a_{i}\right)>n-1
$$

which contradicts (1).

We claim for $a_{i} \in B_{k_{1}}, a_{j} \in B_{k_{2}}, a_{i} \neq a_{j}$,

$$
T_{k_{1}}\left(a_{i}\right) \neq T_{k_{2}}\left(a_{j}\right)
$$

otherwise

$$
p^{h_{i, k_{1}}}\left(a_{i}-p^{k_{1}+1}\right)=p^{h_{j, k_{2}}}\left(a_{j}-p^{k_{2}+1}\right)
$$

hence

$$
a_{i} / p^{k_{1}}-p=a_{j} / p^{k_{2}}-p
$$

which contradicts the $p$-simplicity of $A$.

Now define the following function $F: A \rightarrow \mathrm{N}$ by

$$
F\left(a_{i}\right)= \begin{cases}T_{k}\left(a_{i}\right) & \text { for } a_{i} \in B_{k}, \\ a_{i} & \text { for } a_{i} \in B .\end{cases}
$$

By (9) and (10) $F$ is 1-1. By (2), (4) and (7) we have for all $i=1, \ldots, n$

$$
0<F\left(a_{i}\right) \leq(n-1) p^{d} \text { and } p^{d} \mid F\left(a_{i}\right) \text {. }
$$

This contradiction proves the lemma.

PROOF OF THE THEOREM. We may assume that $A$ is proper, i.e. the g.c.d. of the $a_{i}$ 's is 1 . We may also assume that $p^{d}$ is the lowest $p$-power in $A$ and $d>0$. We suppose

$$
\max _{i, j} \frac{a_{i}}{\left(a_{i}, a_{j}\right)} \leq n-1
$$


Define $B_{0}=\{1,2, \ldots, n-1\} \backslash\left\{p, p^{2}, \ldots, p^{d-1}\right\}$ and $B_{d}=\left\{l p^{d}:\left\lceil n / p^{d}\right\rceil \leq l \leq n-2\right\}$; for $h=1,2, \ldots, d-1$ let

$$
B_{h}=\left\{l p^{h}:\left\lceil n / p^{h}\right\rceil \leq l \leq n-1,(l, p)=1\right\} .
$$

Obviously, $B_{0}, \ldots, B_{d}$ are pairwise disjoint.

We claim

$$
A \subseteq \bigcup_{h=0}^{d} B_{h}
$$

therefore, let $a_{i} \in A$. If $a_{i} \leq n-1$ then $a_{i} \in B_{0}$ since $p^{d}$ is the lowest $p$-power in $A$. If $a_{i} \geq n$ then $p \mid a_{i}$, for otherwise $a_{i} /\left(a_{i}, a_{k}\right)=a_{i} /\left(a_{i}, p^{d}\right)=a_{i} \geq n$, contradicting (11). Now we distinguish between two cases:

Case 1. $p^{h} \| a_{i}$ for some $h, 1 \leq h \leq d-1$. Then $a_{i}=p^{h} a_{i}^{\prime},\left(a_{i}^{\prime}, p\right)=1$, say. Hence $a_{i}^{\prime} \geq\left\lceil n / p^{h}\right\rceil$ and by (11)

$$
p^{h} a_{i}^{\prime}=a_{i} \leq(n-1)\left(a_{i}, a_{k}\right)=(n-1)\left(a_{i}, p^{d}\right)=(n-1) p^{h},
$$

thus $a_{i}^{\prime} \leq n-1$, so $a_{i} \in B_{h}$.

Case 2. $p^{d} \mid a_{i}$. Then $a_{i}=p^{d} a_{i}^{\prime}$, say. Hence $a_{i}^{\prime} \geq\left\lceil n / p^{d}\right\rceil$ and by the lemma $a_{1}<a_{k}=p^{d}$, and by (11)

$$
p^{d} a_{i}^{\prime}=a_{i} \leq(n-1)\left(a_{i}, a_{1}\right) \leq(n-1) a_{1}<(n-1) a_{k}=(n-1) p^{d},
$$

thus $a_{i}^{\prime}<n-1$, so $a_{i} \in B_{d}$.

This proves the claim (12).

We define for $h=1, \ldots, d$

$$
C_{h}=B_{h} \cap A, \quad C_{h}^{\prime}=C_{h} \backslash\left\{(n-1) p^{h}\right\}, \quad C^{\prime}=\bigcup_{h=1}^{d} C_{h}^{\prime} .
$$

Let $B_{0}^{\prime}=\left\{b \in B_{0}: p \nmid b\right\}$. Define a function $f: C^{\prime} \rightarrow B_{0}^{\prime}$ in the following way:

$$
x^{\prime} \in C^{\prime}, x=p^{t} b(p \nmid b, b \leq n-2) \text { say; then } f(x)=b .
$$

The function $f$ is 1-1. Otherwise we have $b=b^{\prime}$ for $x=p^{t} b$ and $x^{\prime}=p^{t^{\prime}} b^{\prime}\left(p \nmid b b^{\prime}\right)$. This implies $p^{t^{\prime}} x=p^{t} x^{\prime}$ which contradicts the $p$-simplicity of $A$.

For $b \in B_{0}^{\prime}$ there is $r$ with $1 \leq r \leq p-1$ and $b \equiv r \bmod p$. Define a function $g: f\left(C^{\prime}\right) \rightarrow B_{0}^{\prime}$ by

$$
g(b)= \begin{cases}b+1 & \text { for } 2 \nmid r \\ b-1 & \text { for } 2 \mid r\end{cases}
$$

Since $2 \mid(p-1)$ and $b \leq n-2$ in (13) we have indeed

$$
g\left(f\left(C^{\prime}\right)\right) \subseteq B_{0}^{\prime}
$$

It is easily seen that $g$ is $1-1$ :

The only interesting case is $g(b)=b+1, g\left(b^{\prime}\right)=b^{\prime}-1, g(b)=g\left(b^{\prime}\right)$. This implies $b=s p+r, b^{\prime}=s^{\prime} p+r^{\prime}$ with $2 \nmid r, 2 \mid r^{\prime}$, for some $s, s^{\prime}$, and $b+2=b^{\prime}$. Hence $\left(s^{\prime}-s\right) p=r-r^{\prime}+2$. Since $\left|r-r^{\prime}\right| \leq p-2$ we can have $p \mid\left(r-r^{\prime}+2\right)$ only for $r-r^{\prime}+2=0$ or $r-r^{\prime}+2=p$. The former gives $r \equiv r^{\prime} \bmod 2$, the latter $r=p-1 \equiv 0 \bmod 2$, both contradicting the conditions on $r$ and $r^{\prime}$. 
For $C=\bigcup_{h=1}^{d} C_{h}$ and $B=\{1,2, \ldots, n-1\}$ define the function $F: C \rightarrow B$ as follows:

$$
F(x)= \begin{cases}g(f(x)) & \text { for } x \in C^{\prime} \\ p^{h} & \text { for } x=(n-1) p^{h}(p \nmid(n-1), 1 \leq h \leq p-1) .\end{cases}
$$

Indeed, $p^{h} \in B$, because $p^{d} \in B$; otherwise, since the g.c.d. of the $a_{i}$ 's is 1 , there is $a_{m}$ with $p \nmid a_{m}$; thus $a_{k} /\left(a_{k}, a_{m}\right)=p^{d} \geq n$ which contradicts (11). By (14) $F$ is 1-1.

For $x \in C^{\prime}, x=p^{t} b(p \nmid b)$, say, we have $p \nmid F(x)$, so $(x, F(x))=\left(p^{t} b, b \pm 1\right)=1$. Since $x \in A, F(x)$ cannot belong to $A$, because $x /(x, F(x)) \geq n$ again contradicts (11). Also, the numbers $p^{h}(1 \leq h \leq d-1)$ do not belong to $A$, because $p^{d}$ is the lowest $p$-power in $A$.

Thus we have found a 1-1 function $F: C \rightarrow B$ with $\operatorname{card}(F(C) \cup(A \cap B))=n$; but $F(C) \cup(A \cap B) \subseteq B$. This contradiction proves the theorem.

\section{REFERENCES}

1. R. D. Boyle, On a problem of R. L. Graham, Acta Arith. 34 (1978), 163-177.

2. E. Z. Chein, On a conjecture of Graham concerning a sequence of integers, Canad. Math. Bull. 21 (1978), 285-287.

3. P. Erdös, Problems and results on combinatorial number theory, A Survey on Combinatorial Theory, North-Holland, Amsterdam, 1973, Chapter 12.

4. R. L. Graham, Unsolved problem 5749, Amer. Math. Monthly 77 (1970), 775.

5. R. Klein, The proof of a conjecture of Graham for sequences containing primes, Proc. Amer. Math. Soc. 95 (1985), 189-190.

6. J. Marica and J. Schönheim, Differences of sets and a problem of Graham, Canad. Math. Bull. 12 (1969), 635-637.

7. R. J. Simpson, On a conjecture of R. L. Graham, Acta Arith. 40 (1982), 209-211.

8. W. Y. Vélez, Some remarks on a number theoretic problem of Graham, Acta Arith. 32 (1977), 233-238.

9. G. Weinstein, On a conjecture of Graham concerning greatest common divisors, Proc. Amer. Math. Soc. 63 (1977), 33-38.

10. R. Winterle, A problem of R. L. Graham in combinatorial number theory, Proc. Louisiana Conf. on Combinatorics, Graph Theory and Computing, Louisiana State Univ., Baton Rouge, La., 1970, pp. 357-361.

INSTITUT FÜR MATHEMATIK, UNIVERSITÄT HANNOVER, WELFENGARTEN 1, 3000 HANNOVER 1, FEDERAL REPUBLIC OF GERMANY 